\title{
¿SUPONE LAMONOPARENTALIDAD UN RIESGO PARA EL RENDIMIENTO ESCOLAR DE LOS HIJOS?
}

\section{Diederik Boertien, Centre d'Estudis Demogràfics}

Con el objetivo de asegurarles un buen futuro, los progenitores siguen varias estrategias para invertir en las habilidades, la educación, las normas y los valores de sus hijos. A ciertas estrategias, como escoger un colegio o leer cuentos a los niños, hay que dedicarles tiempo. Otras, en cambio, requieren que los padres tengan dinero. Algunos ejemplos de estrategias caras son las clases extraescolares o vivir en un "buen" barrio. Por lo tanto, invertir en los hijos es un proceso que exige tiempo y dinero. Los progenitores que viven juntos a menudo pueden ayudarse mutuamente en esta tarea, mientras que hacer todas estas inversiones puede resultar más difícil para los progenitores de familias monoparentales. ¿Deberíamos por ello preocuparnos por las oportunidades que van a tener sus hijos en la vida? ¿Deberíamos preocuparnos por los niños que viven en familias monoparentales de niveles socioeconómicos más bajos? Y, en caso afirmativo, ¿qué podemos hacer al respecto?

En este artículo nos proponemos responder a estas preguntas en relación con España, donde las familias monoparentales femeninas son cada vez más habituales. En primer lugar, utilizamos los datos del censo para documentar el aumento de las familias monoparentales femeninas y mostramos que en 1991 el 6 \% de los niños vivían en una familia monoparental femenina, mientras que en 2011 este porcentaje había crecido hasta el 15 \%. A continuación estudiamos los datos del proyecto PISA de la OCDE para analizar las aptitudes matemáticas de los niños que viven en familias monoparentales. Aunque las puntuaciones en matemáticas de los niños que viven en familias monoparentales son ligeramente inferiores a las de los que viven con dos progenitores, estas diferencias resultan ser muy poco relevantes cuando se examinan más detalladamente. Sostenemos que, si queremos fomentar la igualdad de oportunidades en España, no debemos centrarnos en la estructura familiar sino en otras causas de la desigualdad de oportunidades, como el nivel de ingresos, el barrio y el colegio.

\section{CAMBIOS EN EL PERFIL SOCIOECONÓMICO DE LAS MADRES DE FAMILIAS MONOPARENTALES}

En el pasado, la causa más habitual de que un progenitor terminara criando a sus hijos en solitario era el fallecimiento del padre o la madre. Afortunadamente, hoy en día solo una pequeña minoría de niños vive esta experiencia. La vía más habitual de creación de una familia monoparental en España es actualmente la separación de dos progenitores que estaban casados o vivían juntos. Un reducido porcentaje de niños no llega a vivir nunca con su padre, situación que puede ser planificada o no. Tanto las rupturas entre progenitores como las familias monoparentales femeninas desde el nacimiento han aumentado en España (Castro Martín y Seiz Puyuelo, 2014). Según las cifras del censo, solo el $6 \%$ de los niños menores de 16 años vivían con madre y sin padre en 1991. Este porcentaje subió al 15 \% en 2011.

El perfil de las madres de familias monoparentales también ha ido cambiando. Como se ve en la figura 1, las familias monoparentales femeninas eran ligeramente más habituales entre las mujeres con estudios superiores. Sin embargo, en los últimos años las familias monoparentales femeninas han aumentado especialmente entre las mujeres con menor nivel de estudios (véase también Garriga y Cortina, 2017).

Estos cambios en la relación entre nivel de estudios y familias monoparentales femeninas se han observado en toda Europa. La razón por la que se han producido estos cambios se desconoce. Una teoría clásica sostenía que la separación, mientras no está aceptada socialmente, constituye una opción que solo está al

FIGURA 1. Porcentaje de niños que no viven con un padre en el hogar en función del nivel de estudios de la madre

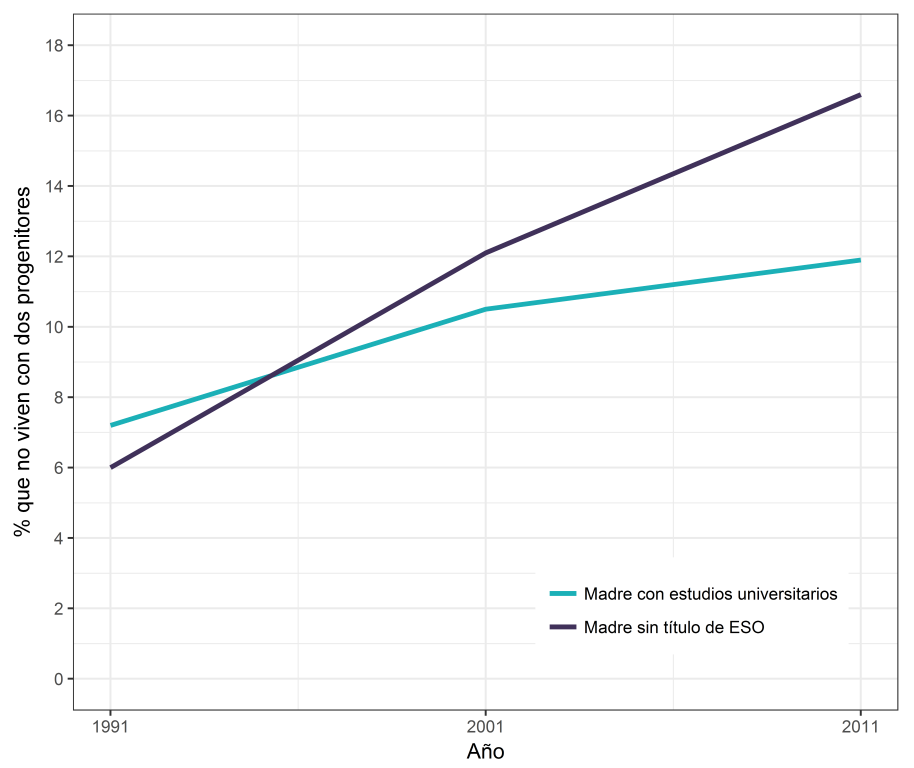

Fuente: Censos españoles de 1991, 2001 y 2011. Niños de 16 años o menos. 
alcance de los más favorecidos de la sociedad (Goode, 1963). En las sociedades "tradicionales", separarse puede ser ilegal, caro o motivo de estigmatización. En un contexto así, separarse puede suponer un paso relativamente rompedor y costoso para los progenitores, por lo que a menudo solo pueden darlo las élites. A medida que la separación pasa a estar más aceptada socialmente, podría volverse más fácil, más barata y ampliamente accesible. En ese caso, se necesitan menos recursos para divorciarse, por lo que las personas de nivel socioeconómico más bajo empiezan a divorciarse también.

En un contexto en el que la mayoría de las personas que desean abandonar a su pareja tienen la posibilidad de divorciarse, la felicidad real de un matrimonio pasa a ser el factor más importante para determinar quién se divorcia y quién no. En consecuencia, se sostiene a menudo que las personas pobres podrían divorciarse más porque están menos satisfechas de sus relaciones. El estrés inducido por factores económicos o las peleas relacionadas con las normas de género pueden ser posibles causas de este menor nivel de satisfacción. No obstante, también es posible que influyan otros motivos más pragmáticos, como el hecho de tener una vivienda en propiedad, que puede convertir la separación en un problema más complejo, en comparación con la situación de las parejas que alquilan una casa conjuntamente.

Independientemente de las razones, la concentración de familias monoparentales entre los grupos desfavorecidos socioeconómicamente puede constituir un obstáculo para el desarrollo de las habilidades y conductas de los hijos de estas familias ¿Hasta qué punto es grave esta cuestión?

\section{RETOS \\ DE LAS \\ MONOPARENTALES \\ FAMILIAS}

La vida de los niños que residen con un solo progenitor es distinta de la vida de los niños que comparten su hogar con dos progenitores. Los niños que viven con un solo progenitor es probable que tengan un contacto menos intensivo con el progenitor que no vive con ellos, mientras que el progenitor que sí convive con los hijos con frecuencia debe asumir en solitario la tarea de criarlos a diario. En muchos casos, ello significa que los progenitores de familias monoparentales, normalmente las madres, tienen que empezar a trabajar menos horas para poder atender a sus hijos, lo que puede conducir a la pobreza u otras causas de estrés económico. Si la familia monoparental es el resultado de una ruptura de los progenitores, los niños también necesitarán adaptarse emocionalmente a la nueva situación. Al mismo tiempo, la separación de los progenitores puede evitar a los niños la exposición a conflictos en el hogar. En conjunto, estos factores pueden constituir obstáculos para que los niños rindan en el colegio, desarrollen sus habilidades, terminen los estudios universitarios y consigan un buen empleo en etapas posteriores de la vida.

Muchos de los retos que plantean las familias monoparentales se pueden minimizar con medidas como, por ejemplo, la custodia compartida, el pago de pensiones de alimentos, el apoyo económico para los progenitores de familias monoparentales y una atención a la infancia de carácter público que pueda facilitar que los progenitores defamilias monoparentales trabajen. La nueva pareja del progenitor también puede ayudar hasta cierto punto en las tareas de crianza, pero su presencia en el hogar también podría requerir una nueva adaptación emocional por parte de los hijos.

En conjunto, un gran número de investigaciones en todo el mundo han documentado la existencia de diferencias en los resultados de los niños en función de la estructura familiar en la que hayan vivido durante su infancia (Härkönen et al., 2017). También es así en España. En la figura 2 se muestran los resultados de distintos grupos de niños en una prueba estandarizada de matemáticas realizada por una muestra amplia y representativa (más de 20.000 niños) de toda España en el marco del programa internacional PISA de la OCDE (http://www.oecd.org/pisa/aboutpisa/).

FIGURA 2. Puntuaciones medias en la prueba de matemáticas de niños de 15 años en España en 2012

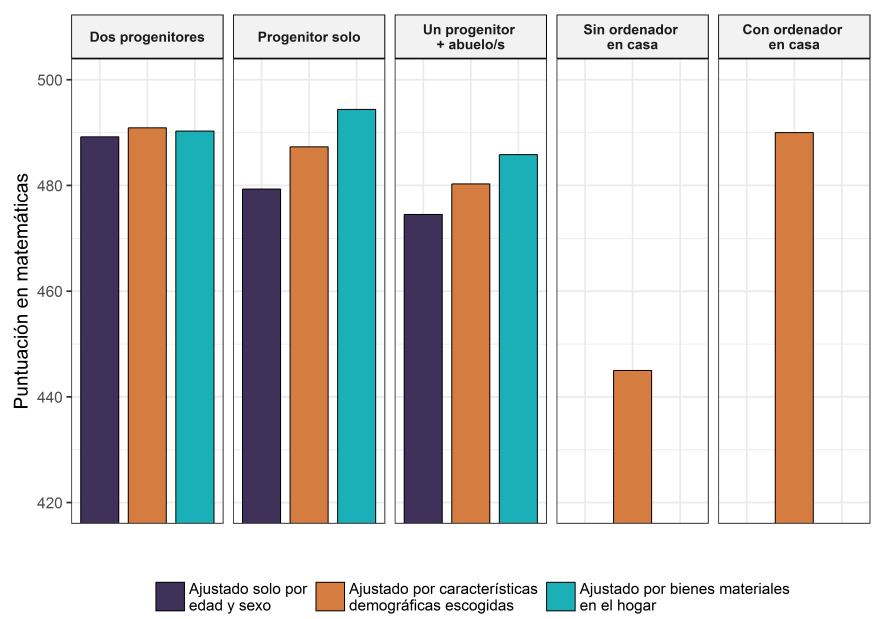

Fuente: PISA 2012; media de cinco puntuaciones en matemáticas posibles

Nota: Progenitor solo: niños que viven con un progenitor y sin ningún abuelo ( 8,5 $\%$ de la muestra). Un progenitor + abuelo/s: niños que viven con un progenitor $y$ como mínimo un abuelo (1,8 \% de la muestra). Las características demográficas son la región de residencia, ser un niño nacido en el extranjero, tener una madre nacida en el extranjero, el sexo del niño, así como el nivel de estudios y la edad de la madre. Bienes materiales en el hogar es un indicador sintético de si el niño tiene acceso en casa a una habitación para él solo, un ordenador o una mesa para estudiar, por ejemplo. Las diferencias entre las barras púrpuras son significativas estadísticamente con un intervalo de confianza del $95 \%$, así como las barras naranjas para la variable de tener un ordenador en casa, no para otras diferencias. 
Puede observarse que los niños que vivían con su padre y su madre obtuvieron una puntuación de 489, mientras que la puntuación de los niños que vivían con un solo progenitor fue de 479 (barras púrpuras de la figura 2). Los niños que vivían con un solo progenitor y, además, uno o más abuelos, obtuvieron puntuaciones aún más bajas: 475 de media. Viendo estas cifras, podría parecer que la estructura familiar influye en los resultados de los niños a lo largo de su vida. Sin embargo, hay importantes reservas que oponer a esta afirmación.

En primer lugar, existe un sinnúmero de características que afectan al rendimiento escolar de los niños. Un objetivo clave de la investigación debería ser detectar cuáles son los factores más importantes que afectan al desarrollo infantil. Por consiguiente, deberíamos comparar las diferencias observadas en función de la estructura familiar con las diferencias observadas en función de otras características. En la figura 2 se muestra cómo difieren las puntuaciones en matemáticas en función de si los niños tienen o no un ordenador en casa. Es probable que carecer de un ordenador en casa sea un indicador de dificultades económicas en el hogar. Los niños sin acceso a un ordenador en casa obtuvieron una puntuación media en la prueba de matemáticas de 445, mientras que los niños que sí tenían un ordenador en casa obtuvieron una puntuación media de 490; la diferencia es de 45 puntos. Esta diferencia es bastante superior a la diferencia de 14 puntos observada en función de la estructura familiar. Por consiguiente, parece ser que la estructura familiar no es tan importante para las habilidades de los hijos como otras características concretas de las familias.

En segundo lugar está la cuestión de la "causalidad". Cuando dos características están relacionadas entre sí, ello no significa automáticamente que una de ellas sea la causa de la otra. Por ejemplo, si las personas pobres tienen más probabilidades de separarse, la obtención de puntuaciones más bajas en matemáticas por parte de los niños que viven con un solo progenitor podría deberse a su situación de pobreza y no a la ausencia de un progenitor en el hogar. Los sociólogos utilizan una gran variedad de estrategias para averiguar si las relaciones entre características se deben a factores adicionales o bien sí reflejan relaciones de causa y efecto. La más sencilla de estas estrategias consiste en analizar las características que ya existían cuando los niños empezaron a vivir en una familia monoparental. Esta estrategia se ha aplicado utilizando algunas características demográficas básicas en las barras naranja oscuro de la figura 2.

Las barras naranja oscuro muestran que las diferencias en el rendimiento escolar en función de la estructura familiar se reducen cuando tenemos en cuenta variables como la región, haber nacido en el extranjero, la edad de la madre y el nivel de estudios de la madre. $\mathrm{Al}$ tener en cuenta estas variables, la puntuación media de los niños que viven con sus dos progenitores pasa a ser solo 4 puntos superior a la puntuación media de los niños que viven con un único progenitor. En el caso de los niños que viven con un progenitor y un abuelo, la diferencia es de 10 puntos. ${ }^{1}$ Probablemente estas diferencias serían aún menores si pudiéramos tener en cuenta más características de las familias, como el estatus socioeconómico de los padres que no viven con sus hijos.

Pese a todo, parece ser que sigue habiendo ciertas diferencias en el rendimiento en matemáticas de los niños que viven con ambos progenitores en comparación con los que no. ¿Cuál podría ser el motivo? A menudo se han propuesto como posibles explicaciones el menor nivel de ingresos y el mayor riesgo de pobreza de los progenitores de familias monoparentales. Los niños que viven con un progenitor y un abuelo podrían estar en una situación de pobreza especialmente acusada. Cuando una familia monoparental se traslada a vivir con los abuelos, un posible motivo es que el progenitor de la familia monoparental no disponga del dinero suficiente para vivir solo con sus hijos.

En España, las diferencias materiales sí parecen ser importantes. Cuando se incluye como variable si los niños tienen acceso en su casa a ciertas cosas, como una habitación para ellos solos, una mesa de estudio o un ordenador, las diferencias de puntuación en las pruebas prácticamente desaparecen, como muestran las barras de color turquesa de la figura 2. Si queremos reducir aún más las diferencias derivadas de la estructura familiar, una buena opción sería invertir en apoyar económicamente a los progenitores de familias monoparentales. Un ejemplo de políticas de este tipo sería mejorar la accesibilidad a la atención de buena calidad a la infancia, lo que permitiría a los progenitores de familias monoparentales dedicar más horas al empleo remunerado.

\section{¿DEBERÍA PREOCUPARNOS LA MONOPARENTALIDAD?}

Ser padre o madre puede ser una experiencia intensa. Todos los progenitores deben enfrentarse a altos niveles de estrés en un momento u otro de la vida de sus hijos. Por ello, no es sorprendente que haya inquietud acerca de si los progenitores de familias monoparentales podrían necesitar ayuda para afrontar la crianza. El aumento del número de familias monoparentales entre las mujeres de menor nivel educativo podría despertar preocupación en particular acerca de la capacidad de sus hijos para cumplir sus objetivos

${ }^{1}$ Si se analizan las pruebas de lengua y ciencias en vez de las de matemáticas, las diferencias resultan ser aún menores o incluso inexistentes. 
en la vida. No obstante, al analizar los resultados reales de los hijos de familias monoparentales, parece ser que los niños que viven con un solo progenitor no tienen un rendimiento muy distinto del de sus iguales.

Existen diferencias en las habilidades cognitivas de los niños en función de su estructura familiar, pero estas diferencias son muy pequeñas en comparación con características como tener un ordenador en casa. Por lo tanto, parece ser que a los niños que viven con un solo progenitor las cosas les van bien. Si nos preocupa la igualdad de oportunidades en la infancia en España, los esfuerzos políticos deberían centrarse en minimizar las diferencias en los resultados de los niños en función de otras características de origen, como los ingresos o el nivel de estudios de los padres.

Para subrayar este punto, la figura 3 presenta un último conjunto de cifras. Las barras de la figura 3 muestran el modo en que las puntuaciones en matemáticas de los niños dependen de los estudios de la madre. Las diferencias son notables y ascienden hasta los 66 puntos, que es nuevamente una diferencia varias veces superior a las observadas entre niños que viven en distintas estructuras familiares. ¿Las brechas debidas al nivel de estudios de la madre serían menores en un mundo en el que todos los niños vivieran con dos progenitores? Es probable que la mejor suposición sobre cómo sería este mundo hipotético sea la basada en la comparación entre los resultados en matemáticas de los niños que en estos momentos sí viven con dos progenitores.

Las barras púrpuras de la figura 3 ofrecen una indicación sobre esta situación hipotética presentando las diferencias en las puntuaciones de las pruebas de matemáticas de PISA en función del nivel de estudios de la madre para el subgrupo de los niños que viven con dos progenitores. Puede observarse que las habilidades matemáticas podrían ser algo superiores en todos los grupos de ese universo imaginario en el que todos los niños viven con dos progenitores. No

Referencias bibliográficas

Castro-Martín, T. ; Seiz-Puyuelo, M. (2014). "La transformación de las familias en España desde una perspectiva socio-demográfica”. VII inform sobre exclusión y desarrollo social en España 2014. Madrid: Fundación FOESSA.

Garriga, A. ; Cortina, C. (2017). "The change in single mothers' educational gradient over time in Spain”. Demographic Research, 36, 1859-1888.

Goode, W.J. (1963). World Revolution and Family Patterns. Nueva York (NY): The Free Press.

Härkönen, J. ; Bernardi, F. ; Boertien, D. (2017). "Family dynamics and child outcomes: An overview of

obstante, las diferencias entre los niños en función del nivel de estudios de la madre cambiarían poco. Entre los niños que viven con su padre y su madre, aquellos cuya madre posee un nivel de estudios bajo obtienen, de media, una puntuación en matemáticas 65 puntos inferior a la de aquellos cuya madre tiene estudios superiores. Esta diferencia es básicamente igual a la observada en el conjunto de la población. En un mundo en el que todos los niños vivieran con sus dos progenitores, es improbable que las diferencias en las habilidades de los niños en función de su situación socioeconómica fueran menores a las actuales.

Si nos preocupan las oportunidades de los niños desfavorecidos en España, no deberíamos centrarnos en las familias monoparentales femeninas ni tampoco en la estructura familiar en general, sino en otros factores que pueden explicar las diferencias en los resultados de los niños. Ejemplos de dichos factores son la variación en los ingresos de las familias, la crianza, los colegios y los barrios.

FIGURA 3. Puntuaciones en matemáticas en función del nivel de estudios de la madre en el conjunto de todas las familias y en aquellas en las que los niños viven con su padre y su madre

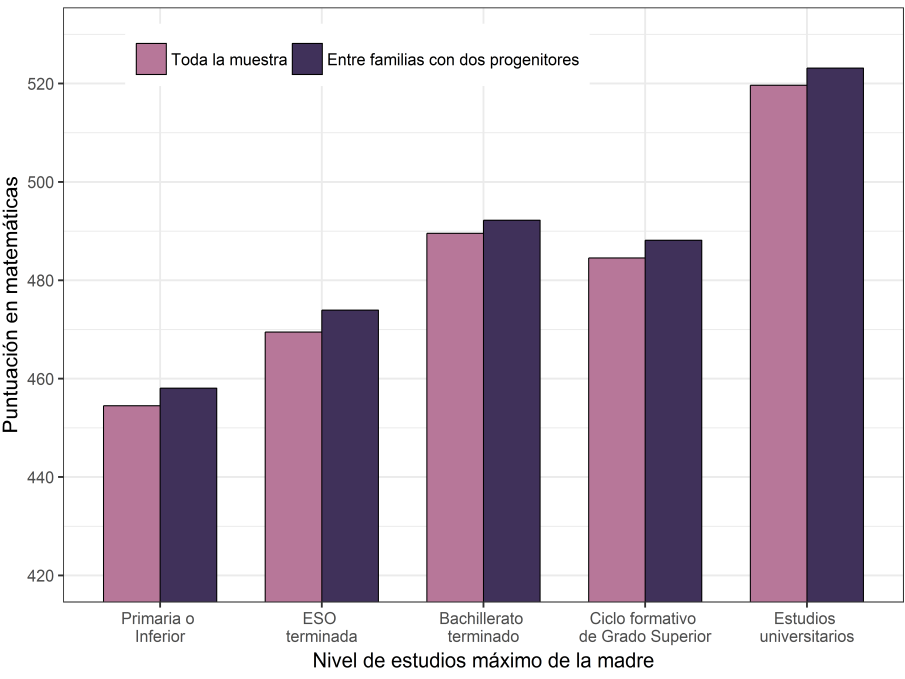

Fuente: PISA 2012; media de cinco puntuaciones en matemáticas posibles.

\section{Agradecimientos}

Varios de los argumentos expuestos en este texto se basan en un trabajo realizado de forma conjunta con $\mathrm{Fa}$ brizio Bernardi del Instituto Universitario Europeo. Deseo expresar mi agradecimiento a Andreu Domingo y Albert Esteve por sus comentarios sobre el contenido. La investigación ha sido posible gracias al programa Beatriu de Pinós de la AGAUR (2016-BPo0121) y al proyecto EQUALIZE del Consejo Europeo de Investigación, liderado por Iñaki Permanyer (ERC2014-StG-637768).
Enlace url

http://ced.uab.es/es/difusion/ butlleti-perspectives-demografiques Contacto

Centre d'Estudis Demogràfics. Calle de Ca n'Altayó, Edificio E2 Universitat Autònoma de Barcelona o8193 Bellaterra / Barcelona España

Teléfono: +34935813060 E-mail: demog@ced.uab.es Web: http://ced.uab.es/es/

\section{UAB}

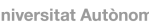

de Barcelona
Gráficos: Anna Turu

Maquetación: Xavier Ruiz Vilchez 